\title{
On Holomorphic Factorization of WZW and Coset Models
}

\section{Edward Witten*}

School of Natural Sciences, Institute for Advanced Study, Olden Lane, Princeton, NJ 08540, USA

Received July 19, 1991

\begin{abstract}
It is shown how coupling to gauge fields can be used to explain the basic facts concerning holomorphic factorization of the WZW model of two dimensional conformal field theory, which previously have been understood primarily by using conformal field theory Ward identities. We also consider in a similar vein the holomorphic factorization of $G / H$ coset models. We discuss the $G / G$ model as a topological field theory and comment on a conjecture by Spiegelglas.
\end{abstract}

\section{Introduction}

The WZW model of two dimensional conformal field theory [1] is a quantum field theory in which the basic field is a map $g: \Sigma \rightarrow G, \Sigma$ being a two dimensional Riemann surface and $G$ being a compact Lie group, which for simplicity we will in this paper take to be simple, connected and simply connected. The basic WZW functional is

$$
I(g)=-\frac{1}{8 \pi} \int_{\Sigma} d^{2} \sigma \sqrt{\varrho} \varrho^{i j} \operatorname{Tr}\left(g^{-1} \partial_{i} g \cdot g^{-1} \partial_{j} g\right)-i \Gamma(g),
$$

where $\varrho$ is a metric on $\Sigma$, $\operatorname{Tr}$ is an invariant form on the Lie algebra of $G$ whose normalization will be specified presently, and $\Gamma$ is the Wess-Zumino term [2]. The latter has the following description [3] in case $\Sigma$ is a Riemann surface without boundary. (For the more general case see [4].) Let $B$ be a three manifold such that $\partial B=\Sigma$, pick an extension of $g$ over $B$, which we will also call $g$, and let

$$
\Gamma(g)=\int_{B} g^{*} \omega=\frac{1}{12 \pi} \int_{B} d^{3} \sigma \varepsilon^{i j k} \operatorname{Tr} g^{-1} \partial_{i} g \cdot g^{-1} \partial_{j} g \cdot g^{-1} \partial_{k} g
$$

where $\omega$ is the left and right invariant three form on the $G$ manifold defined by

$$
\omega=\frac{1}{12 \pi} \operatorname{Tr}\left(g^{-1} d g \wedge g^{-1} d g \wedge g^{-1} d g\right) .
$$

* Research supported in part by NSF Grant PHY86-20266 
$\Gamma(g)$ is well defined (independent of the choice of $B$ and the extension of $g$ over $B$ ) modulo the periods of $\omega$. In these formulas, "Tr" is an invariant quadratic form on the Lie algebra of $G$ which we take to be the smallest multiple of the trace in the adjoint representation such that the periods of $\omega$ are multiples of $2 \pi$. (For $G=S U(N)$, "Tr" is simply the trace in the $N$ dimensional representation.) The condition on the periods ensures that the WZW functional $I(\mathrm{~g})$ is well-defined as a map to $\mathbb{C} / 2 \pi i \mathbb{Z}$, so that $e^{-I(g)}$ is well-defined as a complex valued functional on the space of maps $\Sigma \rightarrow G$.

The Lagrangian of the WZW model is $L(g)=k I(g)$, where $k$ is a positive integer called the "level," and the partition function $Z$ of the WZW model is formally defined as a Feynman path integral,

$$
Z=\int D g e^{-L}=\int D g e^{-k I} .
$$

$Z$ depends, of course, on the metric $\varrho$ of $\Sigma$ which enters in the definition of $I$. Conformal invariance of the WZW model means that apart from a relatively elementary factor given by the conformal anomaly, $Z$ depends only on the complex structure determined by $\varrho$.

The WZW model is essentially exactly soluble; the ability to solve it depends on its holomorphic factorization, first investigated by Knizhnik and Zamolodchikov [5]. Holomorphic factorization of the WZW model means that locally on the space of complex structures one can write $Z=\sum_{i} f_{i} \bar{f}_{i}$, where the $f_{i}$ are holomorphic functions on the space of complex structures. Globally, as advocated by Friedan and Shenker [6], one interprets this formula to mean $Z=(f, f)$, where $f$ is a holomorphic section of a certain flat vector bundle $\mathscr{V}$ over moduli space equipped with a hermitian form ( , ). (The conformal anomaly means that these statements require a somewhat more precise formulation.) The flat bundles that arise in this way have been extensively studied $[7,8]$ and have been seen to have a natural origin in gauge theories [9-12]. To date, the existence of a holomorphic factorization of the WZW model has mostly been understood using conformal field theory Ward identities, this being the original point of view of Knizhnik and Zamolodchikov. The purpose of the present paper is to use gauge theories - or more exactly, coupling of the WZW model to gauge fields - to deduce the existence of a holomorphic factorization. Many of the key steps have been previously exploited by Gawedzki and Kupianen [13, 14]. See also the work of Bernard [15] on the heat equation obeyed by characters of affine Lie algebras. The main novelty which motivated me to write the present paper is the integration over the gauge field and use of the Polyakov-Wiegmann formula to prove that the partition function has a holomorphic factorization; see the derivation of Eq. (2.28). Our treatment will be formal; we will make no claim to analyze the quantum anomalies.

Gauged WZW models have been extensively studied [16-18, 14], mainly with the aim of giving a Lagrangian description of the GKO coset models [19] (whose prehistory goes back to the early days of string theory [20]). After developing our approach to holomorphic factorization of the WZW model in Sect. 2, we will apply the same methods to holomorphic factorization of coset models in Sect. 3 (recovering observations of Moore and Seiberg [21] and Gawedzki and Kupianen $[13,14])$, and then we will consider the special case of the $G / G$ coset model, where sharper statements can be made, as this theory is actually a topological field theory. The $G / G$ model has been investigated by Spiegelglas [22]. 


\section{Gauge Couplings and Holomorphic Factorization}

The WZW action functional $I(g)$ is invariant under the usual action of $G \times G$ (often called $\left.G_{L} \times G_{R}\right)$ on the $G$ manifold. An element $(a, b)$ of $G_{L} \times G_{R}$ acts on $G$ by $g \rightarrow a g b^{-1} .{ }^{1}$ Given a Lagrangian with a (global) symmetry, it is usually possible to "gauge" the symmetry, introducing gauge fields and constructing a gauge invariant extension of the original Lagrangian. In particular, gauging the WZW model means generalizing the theory from the case in which $g$ is a map $\Sigma \rightarrow G$ to the case in which $g$ is a section of a bundle $X \rightarrow \Sigma$ with fiber $G$ and structure group $G_{L} \times G_{R}$ or a subgroup. Letting $A$ be a connection on such a bundle, one aims to find a gauge invariant functional $I(g, A)$ (whose variation with respect to $g$ or $A$ is required to be given by a local formula) which reduces, for $X$ the trivial bundle and $A=0$, to $I(g)$.

In the case of the WZW model, such a gauge invariant extension does not exist. There is no problem in gauging the first term in (1.1) - one just replaces derivatives by covariant derivatives. However, the Wess-Zumino term $\Gamma(g)$ does not have a gauge invariant extension unless one restricts to an "anomaly-free" subgroup $F$ of $G_{L} \times G_{R}$ (and considers bundles $X \rightarrow \Sigma$ with structure group $F$ ). The condition for a subgroup to be anomaly free can be stated as follows. For any subgroup $F$ of $G_{L} \times G_{R}, \mathscr{G}_{L}$ and $\mathscr{G}_{R}$ (the adjoint representations of $G_{L}$ and $G_{R}$ ) are $F$ modules. If $\operatorname{Tr}_{L}$ and $\operatorname{Tr}_{R}$ are the traces in $\mathscr{G}_{L}$ and $\mathscr{G}_{R}$, then the condition for absence of anomalies is that for any $t, t^{\prime} \in \mathscr{F}$ (the Lie algebra of $F$ )

$$
\operatorname{Tr}_{L} t t^{\prime}-\operatorname{Tr}_{R} t t^{\prime}=0 \text {. }
$$

(As will be clear in the appendix, this statement is equivalent to the statement that the class in $H^{3}(G, \mathbb{R})$ represented by $\omega$ has an extension in $H_{F}^{3}(G, \mathbb{R})$, where $H_{F}^{*}$ is the $F$-equivariant cohomology.) In the appendix, we will review the derivation of (2.1) for the benefit of readers not already familiar with such matters and to clarify a few geometrical points. Some readers may want to consult the appendix first, but this should not be necessary for readers who are familiar with gauged WZW models or are willing to verify by hand a few easily verified formulas.

We will also be interested in gauged WZW actions in cases in which (2.1) is not obeyed. In such a case, one cannot construct a gauge invariant $I(g, A)$, but one can find a "best possible" $I(g, A)$, such that the violation of gauge invariance is a multiple of a standard "anomaly" expression that depends on $A$ but not on $g$. [A topological explanation of why it is possible to do this will be given in the appendix, where the detailed formula for $I(g, A)$ is also explained.]

We will adopt the following conventions: $z$ will be a local complex coordinate on $\Sigma, d^{2} z$ is the measure $|d z d \bar{z}|$, and the components of $A$ are defined by $A=A_{z} d z$ $+A_{\bar{z}} d \bar{z}$. We sometimes use the Levi-Civita antisymmetric tensor density $\varepsilon^{i j}$ with $\varepsilon^{z \bar{z}}=-\varepsilon^{\bar{z} z}=i$. (That is, for one forms $a$ and $b$, we write $\int_{\Sigma} a \wedge b=\int_{\Sigma} d^{2} z \varepsilon^{i j} a_{i} b_{j}$ ) Our orientation conventions can be most efficiently and usefully explained by saying that the variation of the Wess-Zumino term under $\delta g=-g u$ is

$$
\delta \Gamma=-\frac{1}{4 \pi} \int_{\Sigma} d^{2} z \varepsilon^{i j} \operatorname{Tr}\left(u g^{-1} \partial_{i} g \cdot g^{-1} \partial_{j} g\right) .
$$

${ }^{1}$ If $G$ has a non-trivial center $Z(G)$, then $Z(G)$, diagonally embedded in $G_{L} \times G_{R}$, acts trivially on $g$, so the faithfully acting symmetry group is really $\left(G_{L} \times G_{R}\right) / Z(G)$. This refinement will not be important until we come to coset models 
For simplicity, we will in this paper consider only the case that $G$ is connected and simply connected, so that a $G$ bundle over $\Sigma$ is trivial.

\subsection{The Holomorphic Wave-Function}

To begin with, we consider the case that $F=G_{R}$. In this case, (2.1) is not obeyed, so a gauge invariant functional $I(g, A)$ does not exist. However, we take

$$
I(g, A)=I(g)+\frac{1}{2 \pi} \int_{\Sigma} d^{2} z \operatorname{Tr} A_{\bar{z}} g^{-1} \partial_{z} g-\frac{1}{4 \pi} \int_{\Sigma} d^{2} z \operatorname{Tr} A_{\bar{z}} A_{z},
$$

which is as close as there is to a gauge invariant functional, in the following sense. Under an infinitesimal gauge transformation,

$$
\delta g=-g u, \quad \delta A_{i}=-D_{i} u=-\partial_{i} u-\left[A_{i}, u\right]
$$

one has

$$
\delta I(g, A)=\frac{1}{4 \pi} \int_{\Sigma} d^{2} z \operatorname{Tr} u\left(\partial_{z} A_{\bar{z}}-\partial_{\bar{z}} A_{z}\right)=\frac{i}{4 \pi} \int_{\Sigma} \operatorname{Tr} u d A
$$

an expression which depends on $A$ but not on $g$ or the complex structure of $\Sigma$. Equation (2.3) is the unique extension of $I(g)$ with this property.

We now formally define a functional of $A$ by

$$
\begin{aligned}
\Psi(A) & =\int D g e^{-k I(g, A)} \\
& =\int D g \exp \left(-k I(g)-\frac{k}{2 \pi} \int_{\Sigma} d^{2} z \operatorname{Tr} A_{\bar{z}} g^{-1} \partial_{z} g+\frac{k}{4 \pi} \int_{\Sigma} d^{2} z \operatorname{Tr} A_{\bar{z}} A_{z}\right) .
\end{aligned}
$$

Note that we do not treat $A$ as a quantum variable; that is, we do not integrate over $A$. This would not be sensible as $I(g, A)$ is not gauge invariant.

Now, $\Psi$ obeys two key equations. First,

$$
\left(\frac{\delta}{\delta A_{z}}-\frac{k}{4 \pi} A_{z}\right) \Psi=0
$$

and second

$$
\left(D_{\bar{z}} \frac{\delta}{\delta A_{\bar{z}}}+\frac{k}{4 \pi} D_{\bar{z}} A_{z}-\frac{k}{2 \pi} F_{\bar{z} z}\right) \Psi=0 .
$$

Equation (2.7) is proved simply by acting with the left-hand side on the integral representation of $\Psi$, and differentiating under the integral sign. Equation (2.8) is a consequence of the standard anomaly Eq. (2.5). By differentiating under the integral sign, one finds that the left-hand side of (2.8) equals

$$
-\frac{k}{2 \pi} \int D g e^{-k I(g, A)}\left[D_{\bar{z}}\left(g^{-1} D_{z} g\right)+F_{\bar{z} z}\right]
$$

where we have introduced covariant derivatives $D g=d g-g A$. The quantity in brackets in (2.9) is the equation of motion of the $g$ field - the variation of $I(g, A)$ under $\delta g=-g u$. Therefore, upon integrating over $g,(2.9)$ vanishes, by integration by parts in $g$ space. 
To elucidate these equations, it is useful to first introduce the operators

$$
\begin{aligned}
& \frac{D}{D A_{z}}=\frac{\delta}{\delta A_{z}}-\frac{k}{4 \pi} A_{\bar{z}} \\
& \frac{D}{D A_{\bar{z}}}=\frac{\delta}{\delta A_{\bar{z}}}+\frac{k}{4 \pi} A_{z} .
\end{aligned}
$$

Note that for $z, w \in \Sigma$,

$$
\left[\frac{D}{D A_{z}(z)}, \frac{D}{D A_{\bar{w}}(w)}\right]=\frac{k}{2 \pi} \delta(z, w) .
$$

In terms of these operators, (2.7) is simply

and (2.8) becomes

$$
\frac{D}{D A_{z}} \Psi=0
$$

$$
\left(D_{\bar{z}} \frac{D}{D A_{\bar{z}}}-\frac{k}{2 \pi} F_{\bar{z} z}\right) \Psi=0,
$$

which in view of (2.12) can be written in a way that does not refer to the complex structure of $\Sigma$ :

$$
\left(D_{i} \frac{D}{D A_{i}}-\frac{i k}{4 \pi} \varepsilon^{i j} F_{i j}\right) \Psi=0 .
$$

These equations are closely related to the basic formulas that appear in canonical quantization of $2+1$ dimensional Chern-Simons gauge theory, as explained, for instance, in Sect. 2 of [11] or in [10]. Let $\mathscr{A}$ be the space of connections on (the trivial $F$ bundle over) $\Sigma . \mathscr{A}$ has a symplectic structure that can be defined purely in differential topology, without choosing a conformal structure on $\Sigma$. It can be defined by the formula

$$
\omega\left(a_{1}, a_{2}\right)=\frac{1}{2 \pi} \int_{\Sigma} \operatorname{Tr} a_{1} \wedge a_{2},
$$

where $a_{1}$ and $a_{2}$ are any two adjoint-valued one forms representing tangent vectors to $\mathscr{A}$. "Prequantization" of $\mathscr{A}$ (in the sense of Kostant [23] and Souriau [24]) means constructing a unitary complex line bundle $\mathscr{L}$ with a connection whose curvature is $-i \omega$. Equation (2.10) can be regarded as a formula defining a connection on the trivial complex line bundle $\mathscr{P}=\mathscr{A} \times \mathbb{C}$ over $\mathscr{A}$ (which we take with the standard unitary structure). This connection according to (2.11) has curvature $-i k \omega$. The factor of $k$ means that $\mathscr{P}$, with this connection, can be identified as $\mathscr{L}^{\otimes k}$, with $\mathscr{L}$ the basic prequantum line bundle.

Hence (2.10) and (2.11) actually describe prequantization of $\mathscr{A}$, with the symplectic structure $k \omega$. The notion of prequantization obviously does not depend on a choice of polarization or complex structure, and indeed, though (2.10) and (2.11) are written in terms of a local complex coordinate on $\Sigma$, they are actually entirely independent of the conformal structure of $\Sigma$. $\Psi(A)$ is best regarded not as a "function" on $\mathscr{A}$ but as a section of the prequantum line bundle $\mathscr{L}^{\otimes k}$.

The complex structure enters when one wishes to quantize $\mathscr{A}$. A choice of complex structure on $\Sigma$ determines a complex structure on $\mathscr{A}$-in which the $A_{\bar{z}}$ are holomorphic and the $A_{z}$ are antiholomorphic. This complex structure is a 
"polarization" which permits quantization: the quantum Hilbert space consists of holomorphic sections of $\mathscr{L}^{\otimes k}$. Equation (2.12) means simply that $\Psi(A)$ is such a holomorphic section.

Now let us discuss the meaning of (2.13). Let $\hat{F}$ be the group of gauge transformations, that is, the group of maps of $\Sigma$ to $F$. Acting on functions on $\mathscr{A}, \hat{F}$ is generated by the operators

$$
D_{i} \frac{\delta}{\delta A_{i}} .
$$

To find a $\hat{F}$ action on sections of $\mathscr{L}^{\otimes k}$, one must "lift" the vector fields in (2.16) appropriately. This can be done in a standard fashion (for instance, see Sect. 2 of [11]); the $\hat{F}$ action on sections of $\mathscr{L}$ is generated by the operators

$$
D_{i} \frac{D}{D A_{i}}-\frac{i k}{4 \pi} \varepsilon^{i j} F_{i j}
$$

(The second term is the contribution of the classical "moment map.") We can thus see the meaning of (2.13) or (2.14) $-\Psi$ is gauge invariant, as a section of $\mathscr{L}^{\otimes k}$.

The two conditions we have found - that $\Psi$ is holomorphic and gauge invariant - mean together that $\Psi$ can be regarded as a physical state of $2+1$ dimensional Chern-Simons gauge theory (with gauge group F). (See [9-11] for more background.) This in fact can be regarded as the essential relation between the WZW model and Chern-Simons theory. We will now recall a few further facts about the Chern-Simons theory. (The facts summarized in the next three paragraphs are not all strictly needed for reading the present paper, but they help put the discussion in context.)

The $\hat{F}$ action on sections of $\mathscr{L}^{\otimes k}$ does not depend on the conformal structure of $\Sigma$, but something new happens once such a conformal structure is picked. A connection $A$ on (the trivial $F$ bundle over) a complex Riemann surface $\Sigma$ determines an operator $\bar{\partial}_{A}$ which defines a complex structure on the bundle. Gauge transformations act by $\bar{\partial}_{A} \rightarrow f \bar{\partial}_{A} f^{-1}$, for $f: \Sigma \rightarrow F$, but as this formula makes sense for $f: \Sigma \rightarrow F_{\mathbb{C}}\left(F_{\mathbb{C}}\right.$ is the complexification of $\left.F\right)$, one actually gets an action of $\hat{F}_{\mathbb{C}}$ (the group of maps of $\Sigma$ to $F_{\mathbb{C}}$ ) on $\mathscr{A}$. A $\hat{F}$ invariant section of $\mathscr{L}^{\otimes k}$ which is also holomorphic is automatically $\hat{F}_{\mathbb{C}}$ invariant. Let $V$ be the space of holomorphic, $\hat{F}_{\mathbb{C}}$ invariant sections of $\mathscr{L}^{\otimes k}$. $V$ is the space of physical states in Chern-Simons gauge theory, at level $k$. From what we have said above, $\Psi$ is a vector in $V$.

A $\widehat{F}$ invariant section of $\mathscr{L}^{\otimes k}$ is the same as a section of an appropriate pushdown line bundle, which we will also call $\mathscr{L}^{\otimes k}$, over the quotient space $\mathscr{A} / \widehat{F}_{\mathbb{C}}$. The quotient $\mathscr{A} / \hat{F}_{\mathbb{C}}$, with the quotient taken in an appropriate sense, is the moduli space of stable holomorphic $F_{\mathbb{C}}$ bundles over $\Sigma$, or (by a theorem of Narasimhan and Seshadri) the moduli space $\mathscr{M}$ of flat $F$ connections on $\Sigma$, up to gauge transformation. This is a compact complex manifold, and in particular, the vector space $V$, which can be identified as $H^{0}\left(\mathscr{M}, \mathscr{L}^{\otimes k}\right)$, is finite dimensional.

So far, when we have made statements that depend on the complex structure of $\Sigma$, we have considered $\Sigma$ with a fixed complex structure. Permitting the complex structure of $\Sigma$ to vary, we get not a single vector space $V$ but a family of vector spaces parametrized by the space $\mathscr{S}$ of complex structures on $\Sigma$, or in short, a vector bundle $\mathscr{V}$ over $\mathscr{S}$. The bundle $\mathscr{V} \rightarrow \mathscr{S}$ has a natural projectively flat connection (which is essential for the topological invariance of Chern-Simons theory); the holomorphic structure is obvious, and the anti-holomorphic structure, which we will recall at an appropriate point, is less obvious. 


\subsection{The Norm of the Wave Function}

By now we have defined, for every complex Riemann surface $\Sigma$, a vector space $V$ consisting of holomorphic, gauge invariant sections of the line bundle $\mathscr{L}^{\otimes k}$ over the space $\mathscr{A}$ of connections. A natural Hermitian structure on $V$ is given formally by

$$
\left(\Psi_{1}, \Psi_{2}\right)=\frac{1}{\operatorname{vol}(\hat{G})} \int_{\mathscr{A}} D A \overline{\Psi_{1}(A)} \Psi_{2}(A) .
$$

(Formally, $D A$ is the measure on $\mathscr{A}$ determined by its symplectic structure, and it is natural to divide by the volume of $\hat{F} \cong \hat{G}$ because of the gauge invariance of $\bar{\Psi}_{1} \Psi_{2}$.) In genus one, this Hermitian structure can be worked out explicitly (that is, reduced to an explicit description of an inner product on the finite dimensional vector space $V$ ), by actually computing the integral over the infinite dimensional $F_{\mathbb{C}}$ orbits $[14,25]$. In genus $>1$, such an explicit evaluation is not known.

We want to compute the norm of the vector $\Psi$ introduced in the last subsection, with respect to this Hermitian structure. To this aim, we first want an integral expression for $\bar{\Psi}$. This could be obtained by just complex conjugating the definition (2.6) of $\Psi$, but instead, we prefer to introduce a conjugate WZW model describing a map $h: \Sigma \rightarrow G$. This time, we introduce a gauge field $B$ gauging the subgroup $G_{L}$ of $G_{L} \times G_{R}$. This is again an anomalous subgroup, so a gauge invariant action $I(h, B)$ extending the WZW action $I(h)$ does not exist. The best that one can do, analogously to (2.3), is

$$
I^{\prime}(h, B)=I(h)-\frac{1}{2 \pi} \int_{\Sigma} d^{2} z \operatorname{Tr} B_{z} \partial_{\bar{z}} h \cdot h^{-1}-\frac{1}{4 \pi} \int_{\Sigma} d^{2} z \operatorname{Tr} B_{z} B_{\bar{z}} .
$$

Under the infinitesimal transformation

one has

$$
\delta h=u h, \quad \delta B_{i}=-D_{i} u,
$$

$$
\delta I^{\prime}(h, B)=-\frac{1}{4 \pi} \int_{\Sigma} d^{2} z \operatorname{Tr} u\left(\partial_{z} B_{\bar{z}}-\partial_{\bar{z}} B_{z}\right)
$$

As in (2.6), we now define

$$
\begin{aligned}
\chi(B) & =\int D h e^{-k I^{\prime}(h, B)} \\
& =\int D h \exp \left(-k I(h)+\frac{k}{2 \pi} \int_{\Sigma} d^{2} z \operatorname{Tr} B_{z} \partial_{\bar{z}} h \cdot h^{-1}+\frac{k}{4 \pi} \int_{\Sigma} d^{2} z \operatorname{Tr} B_{\bar{z}} B_{z}\right) .
\end{aligned}
$$

Comparing (2.6) and (2.22), it is evident that in fact $\chi$ is the complex conjugate of $\Psi$, $\chi(A)=\overline{\Psi(A)}$.

We now come to the key step in the present paper. We use these integral representations to compute $|\Psi|^{2}$ :

$$
\begin{aligned}
|\Psi|^{2}= & \frac{1}{\operatorname{vol}(\hat{G})} \int_{\mathscr{A}} D A \overline{\Psi(A)} \Psi(A) \\
= & \frac{1}{\operatorname{vol}(\hat{G})} \int D g D h D A \exp \left(-k I(g)-k I(h)-\frac{k}{2 \pi} \int_{\Sigma} d^{2} z \operatorname{Tr} A_{\bar{z}} g^{-1} \partial_{z} g\right. \\
& \left.+\frac{k}{2 \pi} \int_{\Sigma} d^{2} z \operatorname{Tr} A_{z} \partial_{\bar{z}} h \cdot h^{-1}+\frac{k}{2 \pi} \int_{\Sigma} d^{2} z \operatorname{Tr} A_{\bar{z}} A_{z}\right)
\end{aligned}
$$


Notice that the integrand is invariant under gauge transformations of the form

$$
\delta g=-g u, \quad \delta h=u h, \quad \delta A_{i}=-D_{i} u .
$$

This follows from the cancellation between (2.5) and (2.21).

We can perform the integral over $A$, using the fact that the exponent in (2.23) is quadratic in $A$ and the operator appearing in the quadratic term is a multiple of the identity. ${ }^{2}$ Gaussian integration over $A$ gives

$$
|\Psi|^{2}=\frac{1}{\operatorname{vol}(\hat{G})} \int D g D h \exp \left(-k I(g)-k I(h)+\frac{k}{2 \pi} \int_{\Sigma} d^{2} z \operatorname{Tr} g^{-1} \partial_{z} g \partial_{\bar{z}} h \cdot h^{-1}\right) .
$$

At this point we may use a formula of Polyakov and Wiegman [26]:

$$
I(g h)=I(g)+I(h)-\frac{1}{2 \pi} \int_{\Sigma} d^{2} z \operatorname{Tr} g^{-1} \partial_{z} g \partial_{\bar{z}} h \cdot h^{-1} .
$$

The proof of this formula follows from the following: (i) the formula is obviously valid if $h=1$; (ii) the left- and right-hand sides are both invariant under $h \rightarrow w h$, $g \rightarrow g w^{-1}$, for arbitrary $w: \Sigma \rightarrow G$. To demonstrate (ii), it suffices to check infinitesimal invariance under $\delta g=-g u, \delta h=u h$. This can easily be verified directly. Actually, a more conceptual proof of (ii) follows from our above calculation. We know that (2.23) is invariant under (2.24), and integrating out $A$, an operation that will preserve this symmetry, one deduces that the exponent on the right-hand side of (2.25) has the desired symmetry.

Therefore, replacing the double integral over $g$ and $h$ by a single integral over $f=g h$, and canceling the factor of $\operatorname{vol}(\hat{G})$ in the process, we get

$$
|\Psi|^{2}=\int D f e^{-k I(f)}
$$

The right-hand side of (2.27) is by definition the partition function $Z(\Sigma)$ of the WZW model (with symmetry group $G$ and level $k$ ) so we have learned

$$
Z(\Sigma)=|\Psi|^{2},
$$

which, though still in need of further elucidation, is the statement of holomorphic factorization of the WZW model.

\subsection{Varying the Complex Structure of $\Sigma$}

So far, we have considered the surface $\Sigma$ with a fixed complex structure. At this level, $Z(\Sigma)$ is a number; $\Psi$ is a vector in a fixed vector space $V$. Equation (2.28) is a relation between them. In this form, the relation is not very remarkable. It gains interest when one permits the complex structure of $\Sigma$ to vary.

We will work over the space $\mathscr{S}$ of all conformal classes of metrics on $\Sigma$. Every conformal metric $\varrho$ determines a complex structure. For any given $\varrho$, we can define a vector space $V_{Q}$ consisting of holomorphic and gauge invariant sections of the

2 We can assume a regularization in which the determinant of a multiple of the identity is one. With an arbitrary regularization, such a determinant is a factor of the form $e^{c \chi(\Sigma)}$, where $c$ is a universal constant, independent of $\Sigma$, and $\chi(\Sigma)$ is the Euler characteristic of $\Sigma$. Such a factor can be removed by adding to the WZW action a multiple of $\int_{\Sigma} \varrho R$, where $R$ is the scalar curvature of a metric $\varrho$ that is compatible with the complex structure of $\Sigma$ 
prequantum line bundle $\mathscr{L}^{\otimes k}$ over $\mathscr{A}$. The $V_{\varrho}$ vary as fibers of a vector bundle $\mathscr{V}$ over $\mathscr{S}$. A section of $\mathscr{V}$ is a function $\Psi(A ; \varrho)$ of connections and conformal metrics which, in its dependence on $A$ for fixed $\varrho$, obeys (2.12) and (2.14).

The space $\mathscr{S}$ is a complex manifold, whose exterior derivative has the standard decomposition $d=\partial+\bar{\partial}$. We will write $\delta^{(1,0)}$ and $\delta^{(0,1)}$ respectively for the $\partial$ and $\bar{\partial}$ operators of $\mathscr{S}$. One can write these explicitly in the form

$$
\begin{aligned}
\delta^{(1,0)} & =\int_{\Sigma} \delta \varrho_{\bar{z} \bar{z} \bar{z}} \frac{\delta}{\delta \varrho_{\bar{z} \bar{z}}}, \\
\delta^{(0,1)} & =\int_{\Sigma} \delta \varrho_{z z} \frac{\delta}{\delta \varrho_{z z}} .
\end{aligned}
$$

The bundle $\mathscr{V}$ has a (projectively) flat structure, which is defined by giving compatibly a holomorphic structure and an antiholomorphic structure. The holomorphic structure is the "obvious" one. $\Psi(A ; \varrho)$ is said to be holomorphic, in its dependence on $\varrho$, if it is annihilated by

$$
\nabla^{(0,1)}=\delta^{(0,1)} .
$$

For the antiholomorphic structure, we cannot simply use the operator $\delta^{(1,0)}$, since this does not commute with the operator on the left-hand side of (2.12). Rather, as explained in $[11,10], \Psi(A ; \varrho)$ is antiholomorphic if it is annihilated by

$$
\nabla^{(1,0)}=\delta^{(1,0)}+\frac{\pi}{2 k} \int_{\Sigma} \delta \varrho_{\bar{z} \overline{\bar{z}}} \operatorname{Tr} \frac{D}{D A_{\bar{z}}} \frac{D}{D A_{\bar{z}}} .
$$

It is now just a matter of differentiating under the integral sign to show that $\Psi(A ; \varrho)$ as defined in $(2.6)$ is annihilated by $\nabla^{(1,0)}$. This has essentially been done in [13]. We have

$$
\delta^{(1,0)} \Psi=\int D g e^{-k I(A, g)}\left(-\frac{k}{8 \pi} \int_{\Sigma} d^{2} z \delta \varrho_{\bar{z} z} \varrho^{\bar{z} z} \operatorname{Tr}\left(g^{-1} D_{z} g\right)^{2}\right),
$$

where $D_{i} g=\partial_{i} g-g A_{i}$. Similarly,

$$
\frac{D}{D A_{\bar{z}}} \Psi=\int D g e^{-k I(g, A)} \cdot \frac{k}{2 \pi} g^{-1} D_{z} g
$$

so that

$$
\operatorname{Tr} \frac{D}{D A_{\bar{z}}} \frac{D}{D A_{\bar{z}}} \cdot \Psi=\int D g e^{-k I(g, A)} \cdot\left(\frac{k}{2 \pi}\right)^{2} \operatorname{Tr}\left(g^{-1} D_{z} g\right)^{2} .
$$

Combining the pieces, we get

$$
\nabla^{(1,0)} \Psi=0
$$

as was claimed.

Now, let $e_{\alpha}, \alpha=1, \ldots, \operatorname{dim} V$ be a basis of orthonormal, covariantly constant sections of $\mathscr{V}$ (over some open set in moduli space). $\Psi$ can be expanded in this basis as

$$
\Psi(A ; \varrho)=\sum_{\alpha} e_{\alpha}(A ; \varrho) \cdot \overline{f_{\alpha}(\varrho)}
$$


with some expansion coefficients $\bar{f}_{\alpha}$. Equation (2.35) means simply that the $\overline{f_{\alpha}(\varrho)}$ are anti-holomorphic functions on $\mathscr{S}$ in the usual sense. Consequently, (2.28) amounts to an expression

$$
Z(\Sigma ; \varrho)=\sum_{\alpha=1}^{\operatorname{dim} V}\left|f_{\alpha}\right|^{2}
$$

for the WZW partition function as a finite sum of norms of holomorphic functions.

The stress tensor of the WZW model is usually defined as

$$
T_{z z}=2 \frac{\delta}{\varrho_{\bar{z} \bar{z}}} I(g, A)=-\frac{k}{4 \pi} \operatorname{Tr}\left(g^{-1} D_{z} g\right)^{2} .
$$

The current is

$$
J_{z}=\frac{\delta}{\delta A_{\bar{z}}} I(g, A)=\frac{k}{2 \pi} g^{-1} D_{z} g
$$

The fact that

$$
T_{z z}=-(\pi / k) \cdot \operatorname{Tr} J_{z}^{2},
$$

which obviously was the main point in the derivation of (2.35), is known as the (classical form of the) Sugawara-Sommerfield construction. It is well known that when $J_{z}$ is defined as a quantum operator, $\operatorname{Tr} J_{z}^{2}$ must be defined with some point splitting or other regularization; this has the effect of replacing $k$ by $k+h$ ( $h$ being the dual Coxeter number of $G$ ). See [13, Eq. (49)] for some discussion of this in the present context.

Obviously, our discussion has been purely formal, and we have made no attempt to prove that the key statements, such as the statement (2.28) of holomorphic factorization, survive the quantum anomalies. A proper treatment would have to address the conformal anomalies that affect both $Z$ and $\Psi$ and show that the left- and right-hand sides of (2.28) have the same conformal anomaly and are equal.

Finally, the gauge invariant functional

$$
\begin{aligned}
I(g, h, A)= & I(g, A)+I^{\prime}(h, A)=I(g)+I(h)+\frac{1}{2 \pi} \int_{\Sigma} d^{2} z \operatorname{Tr} A_{\bar{z}} g^{-1} \partial_{z} g \\
& -\frac{1}{2 \pi} \int_{\Sigma} d^{2} z \operatorname{Tr} A_{z} \partial_{\bar{z}} h \cdot h^{-1}-\frac{1}{2 \pi} \int_{\Sigma} d^{2} z \operatorname{Tr} A_{\bar{z}} A_{z}
\end{aligned}
$$

that appeared in the exponent in (2.23) deserves some comment. Let $G^{\prime}$ be the compact, connected, and simply connected group $G^{\prime}=G \times G$. The pair $(g, h): \Sigma \rightarrow G$ $\times G$ can be regarded as a map of $\Sigma$ to $G^{\prime}$. The $G^{\prime}$ WZW action is just $I(g, h)=I(g)$ $+I(h)$. Let $F$ be the subgroup of $G_{L}^{\prime} \times G_{R}^{\prime}$ consisting of elements of the form $((1, a)$, $\left.\left(a^{-1}, 1\right)\right)$. In other words, $F$ acts by $(g, h) \rightarrow\left(g a^{-1}, a h\right)$. Then $F$ is an anomaly free subgroup of $G_{L}^{\prime} \times G_{R}^{\prime}$ [in the sense that (2.1) is obeyed]. Therefore a gauge invariant action $I(g, h, A)$, reducing to $I(g, h)$ at $A=0$, exists. It is precisely (2.41). Our computation of holomorphic factorization amounted to demonstrating that if $Z_{G}(\Sigma)$ is the partition function of the WZW model with target $G$, and $Z_{G^{\prime} \mid F}(\Sigma)$ is the partition function of a gauged WZW model with target $G^{\prime}$ and gauge group $F$, then

$$
Z_{G}(\Sigma)=Z_{G^{\prime} / F}(\Sigma) \text {. }
$$


Holomorphic factorization has its origin, from this point of view, in the fact that when one computes the action (2.41) of the gauged $G^{\prime} / F$ model, it turns out to be the sum of a functional of $g$ and a functional of $h$. Since exponentiating the action (to get the integrand of the path integral) turns sums into products, this leads to the ability to factorize $Z_{G^{\prime} \mid F}(\Sigma)$ in the fashion that we have described.

\section{Holomorphic Factorization of Coset Models}

So far we have considered gauged WZW models only as a technical tool in order to understand ordinary WZW models. The gauged WZW models are, however, interesting models of conformal field theory in their own right. For every anomalyfree subgroup $F$ of $G_{L} \times G_{R}$ (that is, every subgroup obeying the condition in (2.1)), one has a corresponding gauge invariant generalization of the WZW action, which, upon quantization, leads to a conformal field theory model. The models that arise this way are equivalent to coset models, as has been shown by several authors cited in the introduction.

The most standard examples of anomaly-free subgroups of $G_{L} \times G_{R}$ are the following. Let $G_{\text {adj }}$ be the diagonal subgroup of $G_{L} \times G_{R}$ (acting by $g \rightarrow a g a^{-1}$, $a \in G$ ). Let $H$ be any subgroup of $G_{\text {adj. }}$. Such an $H$ is always anomaly free.

Let $B$ be an $H$-valued connection. Since $H$ is an anomaly-free group, a gaugeinvariant extension $I(g, B)$ of the WZW action $I(g)$ exists. Explicitly, it is

$$
\begin{gathered}
I(g, B)=I(g)-\frac{1}{2 \pi} \int_{\Sigma} d^{2} z \operatorname{Tr} B_{z} \partial_{\bar{z}} g \cdot g^{-1}+\frac{1}{2 \pi} \int_{\Sigma} d^{2} z \operatorname{Tr} B_{\bar{z}} g^{-1} \partial_{z} g \\
-\frac{1}{2 \pi} \int_{\Sigma} d^{2} z \operatorname{Tr}\left(B_{z} B_{\bar{z}}-B_{z} g B_{\bar{z}} g^{-1}\right) .
\end{gathered}
$$

We want to understand the holomorphic factorization of the corresponding coset model partition function

$$
Z_{G / H}(\Sigma)=\frac{1}{\operatorname{vol}(\hat{H})} \int D g D B e^{-k I(g, B)} .
$$

This model (with the diagonal embedding of $H$ in $G_{L} \times G_{R}$ ) is sometimes called "the" $G / H$ model, and corresponds to the diagonal modular invariant, as will be clear. In this paper we will only consider these standard anomaly-free subgroups, but the generalization of the considerations to other cases should be apparent.

\subsection{Holomorphic Wave Function}

As in holomorphic factorization of the original WZW model, we now consider a subgroup $F$ of $G_{L} \times G_{R}$ which is not anomaly free. ${ }^{3}$ In fact, we take $F=H_{L} \times G_{R}$, where $H_{L}$ is the subgroup of $G_{L}$ coming from the embedding of $H$ in $G$. An $F$ connection is a pair $(B, A)$, where $B$ and $A$ are $H$ and $G$ connections, respectively. A

3 The argument could also be expressed in terms of a certain anomaly free subgroup of $G_{L}^{\prime} \times G_{R}^{\prime}$ where $G^{\prime}=G \times G$. This formulation would proceed in parallel with the last paragraph of Sect. 2 
gauge invariant action $I(g, A, B)$ extending the WZW action does not exist, since the subgroup $F$ of $G_{L} \times G_{R}$ is not anomaly free. Analogous to (2.3), there is instead a best possible action, uniquely determined by requiring that the violation of gauge invariance is independent of $g$ and of the conformal structure of $\Sigma$. This action is

$$
\begin{aligned}
I(g, A, B)=I(g) & +\frac{1}{2 \pi} \int_{\Sigma} d^{2} z \operatorname{Tr} A_{\bar{z}} g^{-1} \partial_{z} g-\frac{1}{2 \pi} \int_{\Sigma} d^{2} z \operatorname{tr} B_{z} \partial_{\bar{z}} g \cdot g^{-1} \\
& +\frac{1}{2 \pi} \int_{\Sigma} d^{2} z \operatorname{Tr} B_{z} g A_{\bar{z}} g^{-1}-\frac{1}{4 \pi} \int_{\Sigma} d^{2} z \operatorname{Tr}\left(A_{z} A_{\bar{z}}+B_{z} B_{\bar{z}}\right) .
\end{aligned}
$$

Under

$$
\delta g=v g-g u, \quad \delta A_{i}=-D_{i} u, \quad \delta B_{i}=-D_{i} v
$$

(here $u$ and $v$ are zero forms valued, respectively, in the Lie algebras of $G$ and $H$ ), we have

$$
\begin{aligned}
\delta I(g, A, B) & =\frac{1}{4 \pi} \int_{\Sigma} d^{2} z \operatorname{Tr} u\left(\partial_{z} A_{\bar{z}}-\partial_{\bar{z}} A_{z}-\partial_{z} B_{\bar{z}}+\partial_{\bar{z}} B_{z}\right) \\
& =\frac{i}{4 \pi} \int_{\Sigma} \operatorname{Tr} u(d A-d B) .
\end{aligned}
$$

Before proceeding, let us make a few comments on the global structure. If $G$ has a nontrivial center $Z(G)$, then $Z(G)$, diagonally embedded in $G_{L} \times G_{R}$, acts trivially in the WZW model (since $g=a g a^{-1}$ for $a \in Z(G)$ ). The symmetry group that acts faithfully in the WZW model is hence really $\left(G_{L} \times G_{R}\right) / Z(G)$. Similarly, $F=H_{L} \times G_{R}$ does not act faithfully; the group that acts faithfully is $F^{\prime}=\left(H_{L} \times G_{R}\right) / Z$, where $Z=H \cap Z(G)$. To make the most precise statements in what follows, it is best to think of the pair $(A, B)$ as a gauge field with structure group $F^{\prime}$. The group of maps of $\Sigma$ to $F^{\prime}$ will be called $\hat{F}^{\prime}$. The complexification of $F^{\prime}$ will be called $F_{\mathbb{C}}^{\prime}$, and the group of maps of $\Sigma$ to $F_{\mathbb{C}}^{\prime}$ will be called $\hat{F}_{\mathbb{C}}^{\prime}$. The groups of maps of $\Sigma$ to $H, G$, and their complexifications $H_{\mathbb{C}}$ and $G_{\mathbb{C}}$ will be called $\hat{H}, \hat{G}, \hat{H}_{\mathbb{C}}$, and $\hat{G}_{\mathbb{C}}$.

Now, as in Sect. 2, we introduce the holomorphic wave function

$$
\chi(A, B)=\int D g e^{-k I(g, A, B)} .
$$

$\chi$ obeys certain conditions analogous to those studied in Sect. 2 . To exhibit these, we let $\mathscr{A}$ be the space of $G$-valued connections on $\Sigma, \mathscr{B}$ the space of $H$-valued connections, and $\mathscr{C}=\mathscr{A} \times \mathscr{B}$. We want to consider $\mathscr{C}$ as a symplectic manifold, with the symplectic structure given by the formula

$$
\omega\left(a_{1}, b_{1} ; a_{2}, b_{2}\right)=\frac{1}{2 \pi} \int_{\Sigma} \operatorname{Tr} a_{1} \wedge a_{2}-\frac{1}{2 \pi} \int_{\Sigma} \operatorname{Tr} b_{1} \wedge b_{2} .
$$

(Here the $a_{i}$ and $b_{j}$ are respectively one forms with values in the Lie algebras of $G$ or $H$. The pairs $\left(a_{1}, b_{1}\right)$ and $\left(a_{2}, b_{2}\right)$ define tangent vectors to $\mathscr{C}$. The "Tr" in the second expression on the right of (3.7) is the quadratic form on the $H$ Lie algebra that is induced from the embedding of $H$ in $G$.) The minus sign before the second term in (3.7) is characteristic of coset models. Prequantization means constructing a line bundle $\mathscr{L}$ over $\mathscr{C}$ with a unitary connection of curvature $-i \omega$. 
Rather as in Sect. 2, the line bundle over $\mathscr{C}$ that is relevant is the trivial line bundle endowed with a connection described by the following formulas:

$$
\begin{aligned}
& \frac{D}{D A_{z}}=\frac{\delta}{\delta A_{z}}-\frac{k}{4 \pi} A_{\bar{z}}, \\
& \frac{D}{D A_{\bar{z}}}=\frac{\delta}{\delta A_{\bar{z}}}+\frac{k}{4 \pi} A_{z}, \\
& \frac{D}{D B_{z}}=\frac{\delta}{\delta B_{z}}+\frac{k}{4 \pi} B_{\bar{z}}, \\
& \frac{D}{D B_{\bar{z}}}=\frac{\delta}{\delta B_{\bar{z}}}-\frac{k}{4 \pi} B_{z} .
\end{aligned}
$$

Computing the curvature of this connection, we see that the trivial line bundle endowed with this connection is isomorphic to $\mathscr{L}^{\otimes k}$, which is how we will refer to it henceforth. The action of the gauge group (that is, the group of maps of $\Sigma$ to $G \times H$ ) on $\mathscr{C}$ lifts to an action on $\mathscr{L}^{\otimes k}$. The lift is described at the Lie algebra level by the obvious generalization of $(2.17)$; the $G$ action is generated by the operators

$$
D_{i} \frac{D}{D A_{i}}-\frac{i k}{4 \pi} \varepsilon^{i j} F_{i j}(A),
$$

and the $H$ action is generated by

$$
D_{i} \frac{D}{D B_{i}}+\frac{i k}{4 \pi} \varepsilon^{i j} F_{i j}(B)
$$

Here $F(A)$ and $F(B)$ are the curvatures of $A$ and $B$, respectively. ${ }^{4}$

The analogs of (2.12) and (2.14) are easy to find. $\chi$ obeys first of all

$$
\frac{D}{D A_{z}} \chi=\frac{D}{D B_{\bar{z}}} \chi=0 \text {. }
$$

This has the following interpretation. Pick on $\mathscr{C}$ a complex structure that comes from the standard complex structure on $\mathscr{A}$ and the opposite complex structure on $\mathscr{B}$. (Thus, $A_{\bar{z}}$ and $B_{z}$ are holomorphic, and $A_{z}$ and $B_{\bar{z}}$ are antiholomorphic.) The $(0,2)$ part of the curvature of the connection (3.8) vanishes, so $\mathscr{L}^{\otimes k}$ has a natural structure of holomorphic line bundle over $\mathscr{C}$. Equation (3.11) means that $\chi$ is a holomorphic section of this line bundle. $\chi$ also obeys the analog of (2.14), namely

$$
0=\left(D_{i} \frac{D}{D A_{i}}-\frac{i k}{4 \pi} \varepsilon^{i j} F_{i j}(A)\right) \chi=\left(D_{i} \frac{D}{D B_{i}}+\frac{i k}{4 \pi} \varepsilon^{i j} F_{i j}(B)\right) \chi
$$

As in the discussion of (2.14), this equation means that $\chi$ is gauge invariant in the appropriate sense: it is invariant under the natural lift of the action of the group $\hat{F}^{\prime}$ of gauge transformations to an action on sections of $\mathscr{L}^{\otimes k}$.

${ }^{4}$ If $H$ (or $G$ ) is not connected and simply connected, describing a lift of the gauge group to act on $\mathscr{L}^{\otimes k}$ requires more than the lift of the Lie algebra described by these formulas. The full story is naturally described using the gauge theory approach to prequantization of the space of connections [27-29] and will not be explained here, though the existence of a natural lift is essential later when we consider the role of the center of $G$ 


\subsection{The Space of Conformal Blocks}

Let $W$ be the space of holomorphic sections of $\mathscr{L}^{\otimes k}$ which are $\hat{F}^{\prime}$ invariant - such as $\chi$. We will devote this subsection to a detailed characterization of $W$. $W$ is a finite dimensional vector space which can be given the following concrete description. A holomorphic section of $\mathscr{L}^{\otimes k}$ which is $\hat{F}^{\prime}$ invariant is automatically also $\hat{F}_{\mathbb{C}}^{\prime}$ invariant. Let $\mathscr{R}=\mathscr{C} / \hat{F}_{\mathbb{C}}^{\prime}$. According to the Narasimhan-Seshadri theorem, $\mathscr{R}$ is the moduli space of flat $F^{\prime}$-valued connections on $\Sigma$, up to gauge transformation. $\mathscr{R}$ gets a complex structure from its interpretation as the quotient of the complex manifold $\mathscr{C}$ by the complex group $F_{\mathbb{C}}^{\prime}$. The holomorphic line bundle $\mathscr{L}^{\otimes k}$ over $\mathscr{C}$ pushes down to a holomorphic line bundle, which we will call by the same name, over $\mathscr{R} . \hat{F}_{\mathbb{C}}^{\prime}$ invariant sections of $\mathscr{L}^{\otimes k}$ over $\mathscr{C}$ are pullbacks of sections of $\mathscr{L}^{\otimes k}$ over $\mathscr{R}$, so $W=H^{0}\left(\mathscr{R}, \mathscr{L}^{\otimes k}\right)$. This is the space identified in $[21,14]$ as the space of conformal blocks of the coset model.

$W$ is finite dimensional, since $\mathscr{R}$ is compact. In fact, if $Z$ is trivial, then $\mathscr{R}=\mathscr{M}$ $\times \mathscr{N}$, where $\mathscr{M}=\mathscr{A} / \hat{G}_{\mathbb{C}}$ and $\mathscr{N}=\mathscr{B} / \hat{H}_{\mathbb{C}}$. As is apparent from (3.11), the complex structure on $\mathscr{M}$ is the standard one, and the complex structure on $\mathscr{N}$ is the opposite one. We will refer to $\mathscr{N}$ with the opposite complex structure as $\overline{\mathcal{N}}$. If $\mathscr{L}_{(1)}$ is the standard prequantum line bundle over $\mathscr{M}$ and $\mathscr{L}_{(2)}$ is the standard prequantum line bundle over $\mathscr{N}$ (and we denote their pullbacks to $\mathscr{M} \times \mathscr{N}$ by the same symbols), then $\mathscr{L}^{\otimes k}=\mathscr{L}_{(1)}^{\otimes k} \otimes \mathscr{L}_{(2)}^{\otimes(-k)}$. The minus sign, of course, comes from the minus sign in the second term in (3.7). [As $\mathscr{L}_{(2)}$ has curvature of type $(1,1)$, it is naturally holomorphic both in the standard complex structure on $\mathscr{N}$ and the opposite one.] Consequently, if $Z$ is trivial,

$$
\begin{aligned}
W=H^{0}\left(\mathscr{R}, \mathscr{L}^{\otimes k}\right) & =H^{0}\left(\mathscr{M} \times \bar{N}, \mathscr{L}_{(1)}^{\otimes k} \otimes \mathscr{L}_{(2)}^{\otimes(-k)}\right) \\
& =H^{0}\left(\mathscr{M}, \mathscr{L}_{(1)}^{\otimes k}\right) \otimes H^{0}\left(\bar{N}, \mathscr{L}_{(2)}^{\otimes(-k)}\right) .
\end{aligned}
$$

The space of conformal blocks of the WZW model with target group $G$, studied in Sect. 2, was

$$
V_{G}=H^{0}\left(\mathscr{M}, \mathscr{L}_{(1)}^{\otimes k}\right) \text {. }
$$

Likewise, the space of conformal blocks of the WZW model with target group $H$ is

$$
V_{H}=H^{0}\left(\mathscr{N}, \mathscr{L}_{(2)}^{\otimes k}\right) \text {. }
$$

Here we take $\mathscr{N}$ with its standard complex structure, and a positive tensor power of $\mathscr{L}_{(2)}$. Upon reversing the complex structure on $\mathscr{N}$ and $\mathscr{L}_{(2)}$, we see that, if $V_{H^{*}}$ is the dual vector space to $V_{H}$, then

$$
V_{H^{*}}=H^{0}\left(\overline{\mathcal{N}}, \mathscr{L}^{\otimes(-k)}\right)
$$

Consequently, if $Z$ is trivial,

$$
W=V_{G} \otimes V_{H^{*}}
$$

Now, we want to find the appropriate statement that holds when $Z$ is not trivial. First of all, the natural projection of $F \rightarrow F^{\prime}$ induces a natural map $i: \hat{F} \rightarrow \hat{F}^{\prime} . i$ is not quite an embedding; the kernel consists of constant gauge transformations by elements of the center of $F . i$ is also not quite surjective; the quotient $Z^{\prime}=\hat{F}^{\prime} / i(\hat{F})$ consists of "twists" by elements of $Z$ in going around closed one-cycles in $\Sigma$ (described explicitly at the end of this subsection), so in fact $Z^{\prime}=\operatorname{Hom}\left(H_{1}(\Sigma, \mathbb{Z}), Z\right)$. Thus we have an exact sequence

$$
0 \rightarrow i(\hat{F}) \rightarrow \hat{F}^{\prime} \rightarrow Z^{\prime} \rightarrow 0
$$


Similarly, after complexification (which does not affect finite groups and so leaves $Z$ and $Z^{\prime}$ unmodified), we have a natural projection $i: \widehat{F}_{\mathbb{C}} \rightarrow \widehat{F}_{\mathbb{C}}^{\prime}$ and an exact sequence

with the same $Z^{\prime}$.

$$
0 \rightarrow i\left(\hat{F}_{\mathbb{C}}\right) \rightarrow \hat{F}_{\mathbb{C}}^{\prime} \rightarrow Z^{\prime} \rightarrow 0
$$

We can take the quotient of $\mathscr{C}$ by $\hat{F}_{\mathbb{C}}^{\prime}$ by first dividing by $i\left(\hat{F}_{\mathbb{C}}\right)$ and then dividing by $Z^{\prime}$. As $\mathscr{C} / i\left(\hat{F}_{\mathbb{C}}\right)=\mathscr{M} \times \overline{\mathcal{N}}$, we get a natural action of $Z^{\prime}$ on $\mathscr{M} \times \overline{\mathcal{N}}$, and

$$
\mathscr{R}=(\mathscr{M} \times \bar{N}) / Z^{\prime} \text {. }
$$

From this it follows that, if $X^{Z^{\prime}}$ denotes the $Z^{\prime}$ invariant part of a vector space $X$, then

$$
W=\left(V_{G} \otimes V_{H^{*}}\right)^{Z^{\prime}} .
$$

The $Z^{\prime}$ action on $\mathscr{M} \times \overline{\mathcal{N}}$ that enters here is easy to describe explicitly. According to the Narasimhan-Seshadri theorem, $\mathscr{M} \times \overline{\mathcal{N}}$ is the moduli space of representations of the fundamental group of $\Sigma$ in $G \times H$. For $\Sigma$ of genus $r$, such a representation is given explicitly by holonomies $\left(g_{1}, h_{1}\right), \ldots,\left(g_{2 r}, h_{2 r}\right)$ about $2 r$ generating cycles (modulo conjugation, and subject to a well-known relation). $Z^{\prime}$ acts by $\left(g_{1}, h_{1}\right), \ldots,\left(g_{2 r}, h_{2 r}\right) \rightarrow\left(z_{1} g_{1}, z_{1} h_{1}\right), \ldots,\left(z_{2 r} g_{2 r}, z_{2 r} h_{2 r}\right)$, with $z_{1}, \ldots, z_{2 r}$ being arbitrary elements of $Z$.

\subsection{Holomorphic Factorization}

The vector space $W$ has a natural Hermitian structure formally given by

$$
\left(\chi_{1}, \chi_{2}\right)=\frac{1}{\operatorname{vol}(\hat{G}) \times \operatorname{vol}(\hat{H})} \int_{\mathscr{C}} D A D B \overline{\chi_{1}(A, B)} \chi_{2}(A, B) .
$$

(It is convenient to divide by $\operatorname{vol}(\hat{G}) \cdot \operatorname{vol}(\hat{H})$, and not by $\operatorname{vol}\left(\hat{F}^{\prime}\right)$, which differs from this by a factor of \# $Z^{\prime}$, the number of elements in $Z^{\prime}$.) We want to show that the partition function of the $G / H$ coset model is

$$
Z_{G / H}(\Sigma)=|\chi|^{2} .
$$

The reasoning required is very similar to that in Sect. 2 , so we will be brief. One first introduces a conjugate WZW model, with gauge group $G_{L} \times H_{R}$. The action, for $h: \Sigma \rightarrow G$, and $A$ and $B$ gauge fields of $G_{L}$ and $H_{R}$, is

$$
\begin{aligned}
I^{\prime}(h, A, B)=I(h) & +\frac{1}{2 \pi} \int_{\Sigma} d^{2} z \operatorname{Tr} B_{\bar{z}} h^{-1} \partial_{z} h-\frac{1}{2 \pi} \int_{\Sigma} d^{2} z \operatorname{Tr} A_{z} \partial_{\bar{z}} h \cdot h^{-1} \\
& +\frac{1}{2 \pi} \int_{\Sigma} d^{2} z \operatorname{Tr} A_{z} h B_{\bar{z}} h^{-1}-\frac{1}{4 \pi} \int_{\Sigma} d^{2} z \operatorname{Tr}\left(A_{z} A_{\bar{z}}+B_{z} B_{\bar{z}}\right) .
\end{aligned}
$$

We thus get an integral expression for $\overline{\chi(A, B)}$ :

$$
\overline{\chi(A, B)}=\int D h e^{-k I^{\prime}(h, A, B)} .
$$

Combining (3.6) and (3.25), we get

$$
|\chi|^{2}=\frac{1}{\operatorname{vol}(\hat{G}) \cdot \operatorname{Vol}(\hat{H})} \int D A D B D g D h e^{-k I(g, A, B)-k I^{\prime}(h, A, B)} .
$$


Writing out the exponent on the right-hand side of (3.26) explicitly, one sees that it is quadratic in $A$. The integral over $A$ is Gaussian, therefore. After doing this integral one finds that, using the Polyakov-Wiegman formula, the integral over $g$ and $h$ collapses to an integral over $f=g h$. The remaining functional integral is precisely the definition (3.2) of the partition function $Z_{G / H}(\Sigma)$ of the $G / H$ model, completing the formal proof of (3.23). These steps proceed precisely in parallel with the corresponding points in the derivation of (2.28), and will not be elaborated further.

It remains to consider what happens when the complex structure of $\Sigma$ varies. Again, the parallel with Sect. 2 is so close that we can be brief. When the complex structure of $\Sigma$ varies, $W$ varies, as the fiber of a vector bundle $\mathscr{W}$ over the space $\mathscr{S}$ of complex structures on $\Sigma . \mathscr{W}$ has a projectively flat connection, given by formulas analogous to those of Sect. 2. The holomorphic structure of $\mathscr{W}$ is defined by saying that a section $\chi(A, B ; \varrho)$ is holomorphic if it is annihilated by the $(0,1)$ part of the connection

$$
\nabla^{(0,1)}=\delta^{(0,1)}-\frac{\pi}{2 k} \int_{\Sigma} \delta \varrho_{z z} \operatorname{Tr} \frac{D}{D B_{z}} \frac{D}{D B_{z}} .
$$

The antiholomorphic structure is defined by the $(1,0)$ part of the connection

$$
\nabla^{(1,0)}=\delta^{(1,0)}+\frac{\pi}{2 k} \int_{\Sigma} \delta \varrho_{\bar{z} \bar{z}} \operatorname{Tr} \frac{D}{D A_{\bar{z}}} \frac{D}{D A_{\bar{z}}} .
$$

[The justification of these formulas is that $\nabla$ commutes with the operators on the left hand side of (3.11). Alternatively, one can deduce these formulas systematically by working out the Bogoliubov transformation that compensates for a change in polarization of $\mathscr{C}$. The fact that $B_{z}$ appears in (3.27) and $A_{\bar{z}}$ in (3.28) of course reflects the ubiquitous reversal of sign and change of complex structure of the coset model.] Precisely as in Sect. 2, by differentiating the definition of $\chi$ under the integral sign, one shows that $\chi$ is antiholomorphic,

$$
\nabla^{(1,0)} \chi(A, B ; \varrho)=0 \text {. }
$$

(3.23) and (3.29) make up what is usually called holomorphic factorization of the $G / H$ model.

\section{The $G / G$ Model}

In this section, we will consider the special case of the $G / H$ coset model for $H=G$. This case is particularly simple, being a topological field theory, and as a result sharper statements can be made. The understanding of these statements also illuminates the "ordinary" models, even the original WZW model, as we will see.

The action of the $G / G$ model is the familiar $G / H$ action,

$$
\begin{gathered}
I(g, B)=I(g)-\frac{1}{2 \pi} \int_{\Sigma} d^{2} z \operatorname{Tr} B_{z} \partial_{\bar{z}} g \cdot g^{-1}+\frac{1}{2 \pi} \int_{\Sigma} d^{2} z \operatorname{Tr} B_{\bar{z}} g^{-1} \partial_{z} g \\
-\frac{1}{2 \pi} \int_{\Sigma} d^{2} z \operatorname{Tr}\left(B_{z} B_{\bar{z}}-B_{z} g B_{\bar{z}} g^{-1}\right),
\end{gathered}
$$

specialized to the case $H=G$. Thus, $B$ is now a gauge field valued in the Lie algebra of $G$. ( $B$ is of course gauging the adjoint subgroup of $G_{L} \times G_{R}$, so the covariant 
derivative of $g$ will be $D_{i} g=\partial_{i} g+\left[B_{i}, g\right]$.) The main novelty of the case $G=H$ is that this model is a topological field theory, in the sense that (for instance) the partition function

$$
Z_{G / G}(\Sigma)=\frac{1}{\operatorname{vol}(\hat{G})} \int D B D g e^{-k I(g, B)}
$$

is independent of the metric of $\Sigma$. We will first prove this directly, and then reformulate the argument in the language of holomorphic factorization.

For the direct proof, we note that under an infinitesimal change in the metric of $\Sigma$, the action of the gauged WZW model changes according to the following formula:

$$
\delta I(g, B)=\frac{1}{8 \pi} \int_{\Sigma} \delta \varrho_{\bar{z} \bar{z}} \varrho^{\bar{z} z} \operatorname{Tr}\left(g^{-1} D_{z} g\right)^{2}+\frac{1}{8 \pi} \int_{\Sigma} d^{2} z \delta \varrho_{z z} \varrho^{z \bar{z}} \operatorname{Tr}\left(D_{\bar{z}} g \cdot g^{-1}\right)^{2} .
$$

The variation of the partition function is hence

$$
\begin{gathered}
\delta Z_{G / G}(\Sigma)=\frac{1}{\operatorname{vol}(\hat{G})} \int D B D g e^{-k I(g, B)} \cdot\left(-\frac{k}{8 \pi}\right) \cdot \int_{\Sigma} d^{2} z\left(\delta \varrho_{\bar{z} \bar{z}} \varrho^{\bar{z} z} \operatorname{Tr}\left(g^{-1} D_{z} g\right)^{2}\right. \\
\left.\quad+\int_{\Sigma} d^{2} z \delta \varrho_{z z} \varrho^{z \bar{z}} \operatorname{Tr}\left(D_{\bar{z}} g \cdot g^{-1}\right)^{2}\right)
\end{gathered}
$$

and we must show that this vanishes. To do this, we will show that the integrand in (4.4) is a total derivative in function space. In fact, since the variation of the action in a change of the connection $B$ is

$$
\delta I(g, B)=\frac{1}{2 \pi} \int d^{2} z \operatorname{Tr} \delta B_{\bar{z}} g^{-1} D_{z} g-\frac{1}{2 \pi} \int_{\Sigma} d^{2} z \operatorname{Tr} \delta B_{z} D_{\bar{z}} g \cdot g^{-1},
$$

we get

$$
\begin{aligned}
& \int D B D g \int_{\Sigma} d^{2} z \delta \varrho_{\bar{z} \bar{z}} \varrho^{\bar{z} z} \frac{\delta}{\delta B_{\bar{z}}^{a}} \cdot\left(\left(g^{-1} D_{z} g\right)^{a} \cdot e^{-k I(g, B)}\right) \\
& \quad=\int D B D g e^{-k I(g, B)} \cdot\left(-\frac{k}{2 \pi}\right) \cdot \int_{\Sigma} d^{2} z \delta \varrho_{\bar{z} \bar{z}} \varrho^{\bar{z} z} \operatorname{Tr}\left(g^{-1} D_{z} g\right)^{2}
\end{aligned}
$$

Assuming that one can integrate by part in function space, the left-hand side of (4.6) vanishes, and this means that the first term on the right-hand side of (4.4) can be discarded. The second term on the right-hand side of (4.4) similarly vanishes since

$$
\begin{aligned}
& \int D B D g \int_{\Sigma} d^{2} z \delta \varrho_{z z} \varrho^{z \bar{z}} \frac{\delta}{\delta B_{z}^{a}} \cdot\left(\left(D_{\bar{z}} g \cdot g^{-1}\right)^{a} \cdot e^{-k I(g, B)}\right) \\
& =\int D B D g e^{-k I(g, B)} \cdot\left(\frac{k}{2 \pi}\right) \cdot \int_{\Sigma} d^{2} z \delta \varrho_{z z} \varrho^{z \bar{z}} \operatorname{Tr}\left(D_{\bar{z}} g \cdot g^{-1}\right)^{2} .
\end{aligned}
$$

\subsection{Factorization}

The attentive reader will note that the key fact in the last paragraph was the Sugawara-Sommerfield construction (2.40), which also played a key role in the analysis of holomorphic factorization of general WZW and coset models. In fact, it is illuminating to recast the above argument in the language of holomorphic factorization. 
Precisely as in the general discussion of coset models, we consider the gauging of $H_{L} \times G_{R}$ (which now is $G_{L} \times G_{R}$ ). The closest to a gauge invariant action is (3.17), which we repeat for convenience:

$$
\begin{aligned}
I(g, A, B)=I(g) & +\frac{1}{2 \pi} \int_{\Sigma} d^{2} z \operatorname{Tr} A_{\bar{z}} g^{-1} \partial_{z} g-\frac{1}{2 \pi} \int_{\Sigma} d^{2} z \operatorname{Tr} B_{z} \partial_{\bar{z}} g \cdot g^{-1} \\
& +\frac{1}{2 \pi} \int_{\Sigma} d^{2} z \operatorname{Tr} B_{z} g A_{\bar{z}} g^{-1}-\frac{1}{4 \pi} \int_{\Sigma} d^{2} z \operatorname{Tr}\left(A_{z} A_{\bar{z}}+B_{z} B_{\bar{z}}\right) .
\end{aligned}
$$

The novelty, compared to the case of arbitrary $H$, is that now there is a kind of symmetry between the $G_{L}$ and $G_{R}$ gauge fields $B$ and $A:(4.8)$ is invariant under reversing the complex structure (or equivalently, the orientation) of $\Sigma$, exchanging $g$ with $g^{-1}$, and exchanging $A$ and $B$. [Alternatively, if one exchanges $g$ with $g^{-1}$ and exchanges $A$ and $B$, while leaving the orientation of $\Sigma$ fixed, then (4.8) is complex conjugated.] We want to see the consequences of this.

Just as in the general case of arbitrary $H$, one introduces the holomorphic wave function

$$
\chi(A, B)=\int D g e^{-k I(g, A, B)} .
$$

The general arguments specialized to this case show that the norm of $\chi$ is

$$
|\chi|^{2}=Z_{G / G}(\Sigma)
$$

and that $\chi$ is anti-holomorphic,

$$
\nabla^{(1,0)} \chi=0 .
$$

The novelty is the symmetry between $A$ and $B$, which reverses the complex structures, and so makes it apparent that $\chi$ must also be holomorphic,

$$
\nabla^{(0,1)} \chi=0 .
$$

Equations (4.11) and (4.12) can both be proved by using the general definition (3.27) and (3.28) of the connection and differentiating under the integral sign, as in the proof of (2.35).

Equations (4.11) and (4.12) together mean that $\chi$ is covariantly constant, and hence $|\chi|^{2}$ is a constant. From the factorization law (4.10) we thus deduce again that $Z_{G / G}(\Sigma)$ is independent of the metric.

To probe more deeply, we now recall the general description in Sect. 3 of the vector bundle $\mathscr{W}$ in which holomorphic factorization of the coset model takes place. We had

with

$$
\chi \in\left(V_{G} \otimes V_{H^{*}}\right)^{Z^{\prime}},
$$

$$
\begin{aligned}
V_{G} & =H^{0}\left(\mathscr{M}, \mathscr{L}^{\otimes k}\right), \\
V_{H} & =H^{0}\left(\mathcal{N}, \mathscr{L}^{\otimes k}\right) .
\end{aligned}
$$

Setting $H=G$ and interpreting $V_{G} \otimes V_{G^{*}}$ as $\operatorname{Hom}\left(V_{G}, V_{G}\right)$, we have

$$
\chi \in\left(\operatorname{Hom}\left(V_{G}, V_{G}\right)\right)^{Z^{\prime}} .
$$

Now, $\operatorname{Hom}\left(V_{G}, V_{G}\right)$ contains a canonical (and $Z^{\prime}$-invariant) element, the identity map 1; it is natural to ask whether $\chi=1$. 
The "symmetry" between $A$ and $B$ makes it obvious that $\chi$ is hermitian (in the natural norm on $\left.V_{G}\right)$. Indeed $\chi(A, B)=\overline{\chi(B, A)}$ because (4.8) is complex conjugated if one exchanges $A$ and $B$ while mapping $g \rightarrow g^{-1}$. With our methods it is also easy to prove that

$$
\chi^{2}=\chi \text {. }
$$

This amounts to the statement that

$$
\chi(A, C)=\frac{1}{\operatorname{vol}(\hat{G})} \int D B \chi(A, B) \chi(B, C) .
$$

This is proved by replacing each of the three copies of $\chi$ that appear in (4.17) with the integral representation (4.9), performing the Gaussian integral over $B$, and using the Polyakov-Wiegmann formula (just as in the original proof of holomorphic factorization in Sect. 2.2).

Equation (4.16) means that $\chi$ is the orthogonal projection operator onto a subbundle $\mathscr{V}^{\prime}$ of $\mathscr{V}$ (whose inclusion in $\mathscr{V}$ is compatible with the projectively flat connection on $\mathscr{V}$, since $\chi$ is covariantly constant). It will be evident presently that holomorphic factorization can be carried out in $\mathscr{V}^{\prime}$. One expects that $\chi=1$ and $\mathscr{V}^{\prime}=\mathscr{V}$, but the methods of this paper do not seem to suffice for proving this.

The fact that $\chi$ is covariantly constant means that if $e_{i}(A ; \varrho)$ is an orthonormal basis of covariantly constant sections of $\mathscr{V}^{\prime}$, then $\chi(A, B ; \varrho)=\sum_{i, j} Q_{i j} e_{i}(A ; \varrho) \overline{e_{j}(B ; \varrho)}$, with some constants $Q_{i, j}$. The fact that $\chi^{2}=\chi$ (and $\chi=1$ when restricted to $\mathscr{V}^{\prime}$, by definition of $\mathscr{V}^{\prime}$ ) means that $Q_{i, j}=\delta_{i j}$. So

$$
\chi(A, B ; \varrho)=\sum_{i=1}^{\operatorname{dim} \mathcal{V}^{\prime}} e_{i}(A ; \varrho) \overline{e_{i}(B ; \varrho)} .
$$

We can thus compute the norm of $\chi$ to get

$$
Z_{G / G}(\Sigma)=|\chi|^{2}=\operatorname{dim}\left(\mathscr{V}^{\prime}\right) .
$$

One expects that $\mathscr{V}=\mathscr{V}^{\prime}$, but in any case, if this is not true, it is $\mathscr{V}^{\prime}$ that should be called the space of conformal blocks in the WZW model. (This will be even more apparent in the next subsection.) So we have established that the partition function of the $G / G$ model is the number of conformal blocks of the WZW model, a result that has been conjectured by Spiegelglas [22], with considerable evidence.

\subsection{Relation to the WZW Model and "Ordinary" Coset Models}

Now we will see what we can learn about the original WZW model, and general coset models, by applying our knowledge of the $G / G$ model. The reason that one can learn something interesting is that, upon returning to the definition (4.9) of $\chi$, and noting that $I(g, 0,0)$ is the original action of the WZW model, we see that the partition function of the WZW model is

$$
Z_{G}(\Sigma)=\chi(0,0 ; \varrho) .
$$

In view of (4.18), we get therefore

$$
Z_{G}(\Sigma)=\sum_{i=1}^{\operatorname{dim} \mathscr{V}^{\prime}} e_{i}(0 ; \varrho) \overline{e_{i}(0 ; \varrho)} .
$$


This formula expresses the partition function of the WZW model in terms of quantities that naturally arise in quantizing the moduli space $\mathscr{A}$ of $G$-valued connections, namely the orthonormal parallel sections $e_{i}(A ; \varrho)$.

As a check, let us verify that (4.21) is compatible with the earlier description of $Z_{G}(\Sigma)$ as the norm squared of a holomorphic section of $\mathscr{V}$ :

$$
Z_{G}(\Sigma)=|\Psi|^{2} .
$$

Recalling the definition (2.6) of $\Psi$, we see that $\Psi(A ; \varrho)=\chi(A, 0 ; \varrho)$, so from (4.18) we get

$$
\Psi(A ; \varrho)=\sum_{i=1}^{\operatorname{dim} \mathscr{V}^{\prime}} e_{i}(A ; \varrho) \overline{e_{i}(0 ; \varrho)} .
$$

As the $e_{i}$ are orthonormal, insertion of this in (4.22) gives back (4.21).

In a similar fashion, one can also obtain a formula for the partition function of the $G / H$ model. Recalling the definitions (4.14) of $V_{G}$ and $V_{H}$, we see that there is a natural map $r_{G / H}: V_{G} \rightarrow V_{H}$, which takes a section of $\mathscr{L}^{\otimes k}$ over $\mathscr{M}$ and restricts it to $\mathscr{N}$. (As $\mathscr{M}$ and $\mathscr{N}$ are the moduli spaces of holomorphic $G_{\mathbb{C}}$ and $H_{\mathbb{C}}$ bundles, respectively, there is a natural inclusion of $\mathscr{N}$ in $\mathscr{M}$.) Taking complex conjugates, there is also a natural map $r_{G / H}^{*}: V_{G}^{*} \rightarrow^{H}$. These maps do not respect the unitary structures.

For every $H$, we have holomorphic factorization

$$
Z_{G / H}(\Sigma)=\left|\chi_{G / H}(A, B ; \varrho)\right|^{2},
$$

where $\chi_{G / H}$ is the functional defined in (3.6). Inspecting the definition, we see that

$$
\chi_{G / H}(A, B ; \varrho)=\left(1 \otimes r_{G / H}^{*}\right) \chi_{G / G}(A, B ; \varrho) .
$$

Using (4.18) we can now rewrite (4.24) in the form

$$
Z_{G / H}(\Sigma)=\sum_{i=1}^{\operatorname{dim} \mathscr{V}^{\prime} G}\left|r_{G / H}\left(e_{i}\right)\right|^{2} .
$$

Alternatively,

$$
Z_{G / H}(\Sigma)=\sum_{i=1}^{\operatorname{dim} \mathscr{V}^{\prime} G} \sum_{j=1}^{\operatorname{dim} V_{H}}\left|\left(f_{j}, r_{G / H} e_{i}\right)\right|^{2},
$$

with $f_{j}$ an orthonormal basis of parallel sections of $\mathscr{V}_{H}$. Formulas of this type were suggested in $[13,14]$. If one takes $H$ to be the trivial group (with only the identity element), then (4.26) reduces, as it should, to (4.21).

\section{Appendix}

The purpose of this appendix is to clarify the geometric meaning of the classical gauged WZW actions on which this paper is based. Some readers may wish to consult this appendix before reading the body of the paper (see also $[4,29,30]$ ).

The problem can be clarified by formulating it in more generality than we actually need. We consider an arbitrary connected manifold $M$ with a closed threeform $\omega$ whose periods are multiples of $2 \pi$, so that $\omega$ is related to a class in $H^{3}(M, \mathbb{Z})$. We let $\Sigma$ be an oriented two dimensional surface without boundary. To simplify the considerations that follow, we assume that $\pi_{1}(M)=\pi_{2}(M)=0$, so that a 
continuous map $X: \Sigma \rightarrow M$ is automatically nullhomotopic. (The main novelty that arises if one relaxes this condition is that one must use integral cohomology instead of just working with differential forms.) We suppose given the action of a compact Lie group $F$ on $M$ and we suppose that $\omega$ is $F$ invariant. To simplify the story, we suppose that $F$ is simple and simply-connected. (Again, if these conditions are relaxed, the main novelty that arises is that one must use integral $F$-equivariant cohomology, rather than the de Rham model that will appear presently.) We describe the Lie algebra of $F$ with generators $T_{a}$ and relations

$$
\left[T_{a}, T_{b}\right]=f_{a b}^{c} T_{c}
$$

Let

$$
\Gamma(X)=\int_{B} X^{*} \omega,
$$

where $B$ is any three manifold with $\partial B=\Sigma$, and an arbitrary extension of $X$ over $B$ has been chosen. $\Gamma$ has values in $\mathbb{R} / 2 \pi \mathbb{Z}$. We wish to construct a gauge invariant generalization of $\Gamma$.

The action of $F$ on $M$ is generated by vector fields $V_{a}$. Introducing a gauge field $A=\sum_{a} A^{a} T_{a}$, with structure group $F$, we want to find a generalization $\Gamma(X, A)$ of $\Gamma$ that is invariant under

$$
\delta X=\varepsilon^{a} V_{a}, \quad \delta A^{a}=-D \varepsilon^{a},
$$

for $\varepsilon^{a}$ an infinitesimal gauge transformation. The variation of $\Gamma$ is

$$
\delta \Gamma=\int_{\Sigma} \varepsilon^{a} X^{*}\left(i_{V_{a}}(\omega)\right) .
$$

$\left(i_{V}\right.$ is the operation of contracting with a vector field $V$.) Additional terms that can be added to (A.2) to cancel this exist only if there are one-forms $\lambda_{a}$ on $M$ such that

$$
i_{V_{a}}(\omega)=d \lambda_{a},
$$

and moreover such that

$$
i_{V_{a}}\left(\lambda_{b}\right)+i_{V_{b}}\left(\lambda_{a}\right)=0 .
$$

If such $\lambda_{a}$ exist, then, by averaging suitably over the compact group $F$, one can suppose that they transform in the adjoint representation of $F$. In this case, the desired gauge invariant generalization of $\Gamma$ is

$$
\Gamma(X, A)=\Gamma(X)-\int_{\Sigma} A^{a} \wedge X^{*}\left(\lambda_{a}\right)-\frac{1}{2} \int_{\Sigma} A^{a} \wedge A^{b} \cdot X^{*}\left(i_{V_{b}} \lambda_{a}\right) .
$$

Equations (A.5) and (A.6) have a geometrical meaning, in terms of the so-called $F$-equivariant cohomology of $M$, denoted $H_{F}^{*}(M)$. A de Rham model for this equivariant cohomology, explained in [31, 32], can be described as follows. Let $\Omega^{*}(M)$ be the de Rham complex of $M$, and let $S^{*}(\mathscr{F})$ be a symmetric algebra on the Lie algebra $\mathscr{F}$ of $F$, with generators $\phi^{a}$ considered to be of degree two. Let $W^{*}=\left(\Omega^{*}(M) \otimes S^{*}(\mathscr{F})\right)^{F}$ (with ${ }^{F}$ denoting the $F$ invariant part). In $W^{*}$, introduce the differential

$$
D=d+\sum_{a} \phi^{a} i_{V_{a}} .
$$


If $\omega$ is a closed form on $M$, an element $\bar{\omega} \in W^{*}$ is called an equivariant extension of $\omega$ if $D \bar{\omega}=0$ and $\left.\bar{\omega}\right|_{\phi=0}=\omega$. The meaning of (A.5) and (A.6) is simply that they are the conditions for $\omega$ to have an equivariant extension. In fact,

$$
\bar{\omega}=\omega-\sum_{a} \phi^{a} \lambda_{a}
$$

is an equivariant extension of $\omega$ if and only if the $\lambda_{a}$ obey (A.5) and (A.6) and transform in the adjoint representation of $F$.

Now let us specialize to the case of actual interest in this paper in which $M$ is the group manifold of a simple, compact, connected, and simply-connected Lie group $G$, and

$$
\omega=\frac{1}{12 \pi} \operatorname{Tr}\left(g^{-1} d g\right)^{3} .
$$

Moreover, $F$ is a connected subgroup of $G_{L} \times G_{R}$. The embedding of $F$ in $G_{L}$ and $G_{R}$ is determined by an embedding of Lie algebras which we can write as

$$
T_{a} \rightarrow\left(T_{a, L}, T_{a, R}\right) .
$$

The vector fields $V_{a}$ are described by the formula

$$
\delta g=\sum_{a} \varepsilon^{a}\left(T_{a, L} g-g T_{a, R}\right) .
$$

One has

with

$$
i_{V_{a}} \omega=d \lambda_{a}
$$

$$
\lambda_{a}=\frac{1}{4 \pi} \operatorname{Tr}\left(T_{a, L}\left(d g \cdot g^{-1}\right)+T_{a, R}\left(g^{-1} d g\right)\right) .
$$

These $\lambda_{a}$ transform in the adjoint representation of $F$. The non-uniqueness in the choice of the $\lambda_{a}$ is $\lambda_{a} \rightarrow \lambda_{a}+d w_{a}$, where the $w_{a}$ are zero forms in the adjoint representation of $F$. Equation (A.14) is the unique universal formula that works for any $F$. One now computes that

$$
i_{V_{a}}\left(\lambda_{b}\right)+i_{V_{b}}\left(\lambda_{a}\right)=\frac{1}{2 \pi} \operatorname{Tr}\left(T_{a, L} T_{b, L}-T_{a, R} T_{b, R}\right) .
$$

[Note that the possible $w_{a}$ do not contribute since $i_{V_{a}}\left(d w_{b}\right)=f_{a b}^{c} w_{c}$ is antisymmetric in $a$ and $b$.] Thus the equivariant extension $\bar{\omega}$ of $\omega$ and the corresponding gauge invariant extension $\Gamma(\mathrm{g}, A)$ of $\Gamma$ exist precisely if $F$ is such that the right-hand side of (A.15) vanishes.

This is the criterion that was stated in (2.1). The gauge invariant extension of $\Gamma$, when $F$ is such that (A.15) vanishes, is explicitly

$$
\begin{aligned}
\Gamma(g, A)=\Gamma(g)-\frac{1}{4 \pi} \int_{\Sigma} A^{a} \wedge \operatorname{Tr}\left(T_{a, L} d g \cdot g^{-1}+T_{a, R} g^{-1} d g\right) . \\
-\frac{1}{8 \pi} \int A^{a} \wedge A^{b} \operatorname{Tr}\left(T_{a, R} g^{-1} T_{b, L} g-T_{b, R} g^{-1} T_{a, L} g\right) .
\end{aligned}
$$

Even when (A.15) does not vanish, (A.16) is the closest that there is to a gauge invariant extension of $\Gamma(\mathrm{g}, A)$, in the sense that the variation of (A.16) under a gauge transformation depends only on $A$ and is independent of $g$. This fact, which played an important role in the body of the paper, reflects the fact that the $\lambda_{a}$ obeying (A.5) 
exist for any $F$; only the validity of (A.6) depends on $F$. This means that although an extension $\bar{\omega}$ of $\omega$ obeying $D \bar{\omega}=0$ may not exist, $\omega$ always has an extension such that

$$
D \bar{\omega} \in S^{*}(\mathscr{F}) .
$$

(And such an $\bar{\omega}$ is unique if one wishes a formula that works universally for any $F$.) This relation precisely ensures that the violation of gauge invariance depends on $A$ and not $g$.

Geometrically, the reason that (A.17) has a solution is as follows. The equivariant cohomology of $G$ is the cohomology of the homotopy quotient $G / / F$ $=G \times{ }_{F} E F$. If one computes the cohomology of $G / / F$ from the spectral sequence of the fibration $G / / F \rightarrow B F$, one sees (since $\omega$ is a three dimensional class, and the nontrivial cohomology of $B F$ begins in dimension four) that the only obstruction to existence of an equivariant extension $\bar{\omega}$ of $\omega$ comes from $H^{4}(B F)$. In fact, the invariant quadratic form on the $F$ Lie algebra that appears on the right-hand side of (A.15) represents the obstruction class in $H^{4}(B F)$, via the Chern-Weil homomorphism. The cohomology of $B F$ is isomorphic to $S^{*}(\mathscr{F})$, so the obstruction is an element of $S^{*}(\mathscr{F})$.

The gauge invariant generalization of the WZW Lagrangian is

$$
I(g, A)=-\frac{1}{8 \pi} \int_{\Sigma} \operatorname{Tr} g^{-1} d_{A} g \wedge * g^{-1} d_{A} g-i \Gamma(g, A),
$$

with $*$ the Hodge star operator, $d_{A}$ the gauge-covariant extension of the exterior derivative, and $\Gamma(g, A)$ given in (A.16). The first term depends on the conformal structure of $\Sigma$, and the second has a topological origin that we have attempted to elucidate in this appendix. The properties of the WZW model depend on a peculiar interplay between the two terms, some aspects of which we have seen in this paper. All the particular formulas for gauged WZW Lagrangians given in this paper are various specializations of (A.18).

\section{References}

1. Witten, E.: Non-Abelian bosonization in two dimensions. Commun. Math. Phys. 92, 455 (1984)

2. Wess, J., Zumino, B.: Consequences of anomalous ward identities. Phys. Lett. 37 B, 95 (1971)

3. Witten, E.: Global aspects of current algebra. Nucl. Phys. B 223, 422 (1983)

4. Felder, G., Gawedzki, K., Kupianen, A.: Spectra of Wess-Zumino-Witten models with arbitrary simple groups. Commun. Math. Phys. 117, 127 (1988)

Gawedzki, K.: Topological actions in two-dimensional quantum field theories. In: Nonperturbative quantum field theory. 't Hooft, G. et al. (eds.). London: Plenum Press 1988

5. Knizhnik, V.G., Zamolodchikov, A.B.: Current algebra and Wess-Zumino model in two dimensions. Nucl. Phys. B 247, 83 (1984)

6. Friedan, D., Shenker, S.: The analytic geometry of two-dimensional conformal field theory. Nucl. Phys. B 281, 509-545 (1987)

7. Verlinde, E.: Fusion rules and modular transformations in $2 \mathrm{~d}$ conformal field theory. Nucl. Phys. B 300, 351

8. Moore, G., Seiberg, N.: Polynomial equations for rational conformal field theories. Phys. Lett. B 212, 360 (1988); Classical and quantum conformal field theory. Nucl. Phys. B

9. Witten, E.: Quantum field theory and the Jones polynomial. Commun. Math. Phys. 121, 351 (1989)

10. Elitzur, S., Moore, G., Schwimmer, A., Seiberg, N.: Remarks on the canonical quantization of the Chern-Simons-Witten theory. IAS preprint HEP-89/20 
11. Axelrod, S., DellaPietra, S., Witten, E.: Geometric quantization of Chern-Simons gauge theory. J. Diff. Geom. 33, 787 (1991)

12. Hitchin, N.: Flat connections and geometric quantization. Commun. Math. Phys. 131, 347 (1990)

13. Gawedzki, K.: Constructive conformal field theory. In: Functional integration, geometry, and strings. Hava, Z., Sobczyk, J. (eds.). Boston, Basel: Birkhäuser 1989

14. Gawedzki, K., Kupianen, A.: $G / H$ conformal field theory from gauged WZW model. Phys. Lett. 215B, 119 (1988); Coset construction from functional integrals. Nucl. Phys. B 320 (FS), 649 (1989)

15. Bernard, D.: On the Wess-Zumino-Witten models on the torus. Nucl. Phys. B 303, 77 (1988); On the Wess-Zumino-Witten models on Riemann surfaces. Nucl. Phys. B 309, 145 (1988)

16. Guadagnini, E., Martellini, M., Minchev, M.: Phys. Lett. 191 B, 69 (1987)

17. Bardacki, K., Rabinovici, E., Saring, B.: Nucl. Phys. B 299, 157 (1988)

Altschuler, A., Bardacki, K., Rabinovici, E.: Commun. Math. Phys. 118, 241 (1988)

18. Karabali, D., Park, Q.-H., Schnitzer, H.J., Yang, Z.: Phys. Lett. 216B, 307 (1989)

Schnitzer, H.J.: Nucl. Phys. B 324, 412 (1989)

Karabali, D., Schnitzer, H.J.: Nucl. Phys. B 329, 649 (1990)

19. Goddard, P., Kent, A., Olive, D.: Phys. Lett. B 152, 88 (1985)

20. Bardacki, K., Halpern, M.B.: Phys. Rev. D 3, 2493 (1971)

Halpern, M.B.: Phys. Rev. D 4, 2398 (1971)

21. Moore, G., Seiberg, N.: Taming the conformal zoo. Phys. Lett. B

22. Spiegelglas, M.: Lecture at IAS (October, 1990), Setting Fusion Rules in Topological LandauGinzburg. Technion preprint; Spiegelglas, M., Yankielowicz, S.: $G / G$ Topological Field Theory by Cosetting. Fusion Rules As Amplitudes in $G / G$ Theories. Preprints (to appear)

23. Kostant, B.: Orbits, symplectic structures, and representation theory, Proc. of the U.S.-Japan Seminar in Differential Geometry (Kyoto, 1965); Quantization and Unitary Representations. Lecture Notes in Math., vol. 1170, p. 87. Berlin, Heidelberg, New York: Springer 1970; Line Bundles and the Prequantized Schrodinger Equation. Coll. Group Theoretical Methods in Physics (Marseille, 1972) p. 81

24. Souriau, J.: Quantification geometrique. Commun. Math. Phys. 1, 374 (1966). Structures des systemes dynamiques. Paris: Dunod 1970

25. Gawedzki, K.: Quadrature of conformal field theories. Nucl. Phys. 328, 733 (1989)

26. Polyakov, A.M., Wiegman, P.B.: Theory of non-abelian Goldstone bosons in two dimensions. Phys. Lett. B 131, 121 (1983)

27. Ramadas, T.R., Singer, I.M., Weitsman, J.: Some comments on Chern-Simons gauge theory. MIT preprint

28. Freed, D.: Preprint (to appear)

29. Axelrod, S.: Ph. D. thesis, Princeton University (1991), Chapter four

30. Hull, C.M., Spence, B.: The geometry of the gauged sigma model with Wess-Zumino Term. Queen Mary and Westfield College preprint QMW 90/04

31. Atiyah, M.F., Bott, R.: The moment map and equivariant cohomology. Topology 23, 1 (1984)

32. Mathai, V., Quillen, D.: Superconnections, Thom classes, and equivariant differential forms. Topology 25, 85 (1986)

Communicated by A. Jaffe 